\title{
Sonographisches Bildessay: Periphere Nerventumoren
}

\author{
N. Bendix \\ C. Wolf \\ H. Gruber \\ G. Bodner
}

\section{Pictorial Essay: Ultrasound of Tumours and Tumour-like \\ Lesions of Peripheral Nerves}

\section{Zusammenfassung}

Periphere Nerven und von peripheren Nerven ausgehende Raumforderungen lassen sich durch die hochauflösende und hochfrequente Sonographie exzellent darstellen. Mit Breitbandschallköpfen von zwischen 5 und $15 \mathrm{MHz}$ erkennt man auch kleine Nerven, die echoarmen Nervenfaszikel und das umgebende echoreiche Bindegewebe sowie nervale Raumforderungen und die Beziehung zu ihrem Ursprungsnerv. Zusätzlich lässt sich mit der farbkodierten Dopplersonographie eine vorhandene Gefäßversorgung der Tumoren erkennen und beurteilen. In dieser Arbeit beschreiben wir das sonographische Erscheinungsbild von benignen und malignen Tumoren peripherer Nerven.

Schliusselwörter

Ultraschall · Periphere Nerventumore

\section{Abstract}

High-resolution ultrasound is an excellent diagnostic modality for visualising peripheral nerves and differentiate tumours of the peripheral nerve. With high-frequency transducers (5-15 $\mathrm{MHz}$ ), peripheral nerves can be visualised, the hypoechoic nerve fascicles, the surrounding tissue and tumourous lesions including the relation to their nerve of origin can be evaluated. By using colour Doppler sonography (CDS), it is possible to detect and assess vascularisation of tumours of the nerve. We present sonographic features of common and uncommon benign and malignant tumours of the peripheral nerve.

Key words

Ultrasound · tumours of peripheral nerve

\section{Einleitung}

Bereits aus den 80er-Jahren stammen die ersten Arbeiten über den Einsatz der Sonographie zur Darstellung von peripheren Nerven sowie von Nerventumoren.

Fornage gab 1988 erstmals einen Überblick über die sonographische Untersuchung peripherer Nerven [1].
Durch die heutige Verfügbarkeit hochfrequenter und hochauflösender Ultraschall-Geräte lassen sich periphere Nerven sehr gut darstellen.

Gegenüber anderen Untersuchungsmodalitäten und Schnittbildverfahren ist es durch die Sonographie möglich, in einem Untersuchungsgang große anatomische Abschnitte zu erfassen und somit auch periphere Nerven in ihrem Verlauf darzustellen,

Institutsangaben

Klinische Abteilung für Radiodiagnostik 1, Universitätsklinik für Radiodiagnostik, Medizinische Universität Innsbruck

Korrespondenzadresse

Dr. N. Bendix · Medizinische Universität Innsbruck, Universitätsklinik für Radiodiagnostik, Klinische Abteilung für Radiodiagnostik 1 · Anichstraße 35 · A-6020 Innsbruck · Tel.: ++ 43/512/50422761 .

Fax: ++ 43/512/50422758 ·E-mail: nadine.bendix@uibk.ac.at

eingereicht: $25.10 .2004 \cdot$ angenommen: 4.3 .2005

Bibliografie

Ultraschall in Med 2005; 26: 318 - 324 @ Georg Thieme Verlag KG Stuttgart · New York

DOI 10.1055/s-2005-858374

ISSN 0172-4614 
Raumforderungen zu erkennen sowie die Beziehung zu ihrem Ursprungsgewebe zu lokalisieren, unter anderem auch durch die Darstellbarkeit in verschiedenen Ebenen.

Ein weiterer großer Vorteil der Sonographie besteht im Einsatz des Farbdopplers zur Beurteilung einer eventuell vorhandenen Gefäßversorgung.

Im Folgenden wird das sonographische Erscheinungsbild von häufigen und seltenen Nerventumoren beschrieben.

\section{Amputationsneurom, posttraumatisches Neurom}

Kommt es im Rahmen der Schädigung eines peripheren Nervs zur Kontinuitätsunterbrechung von Axonen, geht nach einigen Tagen der gesamte distale Axonabschnitt im Rahmen der so genannten Waller-Degeneration zugrunde. Schwann-Zellen und eingewanderte Histiozyten resorbieren die distalen Axone und ihre zerfallenen Markscheiden.

Im weiteren Verlauf des Regenerationsprozesses peripherer Nerven proliferieren die Schwann-Zellen des proximalen und distalen Axonabschnittes und bilden so genannte Büngner'sche Bänder, die zur Orientierung der bereits wenige Tage nach Kontinuitätsunterbrechung aussprossenden Axone des proximalen Nervenabschnittes dienen sollen. Gelingt der Anschluss an diese Pfade aus Zellsäulen, können die Axone in ihr ursprüngliches Terminalgebiet auswachsen und werden schließlich von distal nach proximal hin remyelinisiert [2].

Ist der distale Axonabschnitt nach einer Amputation allerdings nicht mehr vorhanden, kommt es durch Proliferation aller Strukturkomponenten im Rahmen des Regenerationsprozesses des peripheren Nervs zur Ausbildung eines Amputationsneuromes am proximalen Nervenende.

Im Rahmen traumatischer Schädigungen peripherer Nerven kann es neben dieser kompletten Kontinuitätsunterbrechung durch weitere auf den Nerv einwirkende Kräfte zur partiellen Schädigung auch weiter proximal gelegener Abschnitte kommen, so dass sich hier so genannte Traktionsneurome bilden können.

Mithilfe der hochauflösenden Sonographie können Neurome sehr gut detektiert werden. Sie stellen sich als knotige, umschriebene und echoarme Knoten dar, die kontinuierlich aus dem geschädigten Nerv hervorgehen. Häufig kommen die Nervenfaszikel des Nervenendes aufgetrieben zur Darstellung, im Inneren des Neuromes selbst können noch einzelne Nervenfaszikel erkennbar werden (Abb.1).

Im Verlauf eines partiell geschädigten Nervs kann es zur Entwicklung mehrerer Neurome, der so genannten Traktionsneurome, innerhalb der erhaltenen Nervenscheide kommen (Abb. 2).

Farbdopplersonographisch weisen Neurome keine Vaskularisierung auf [3-8].

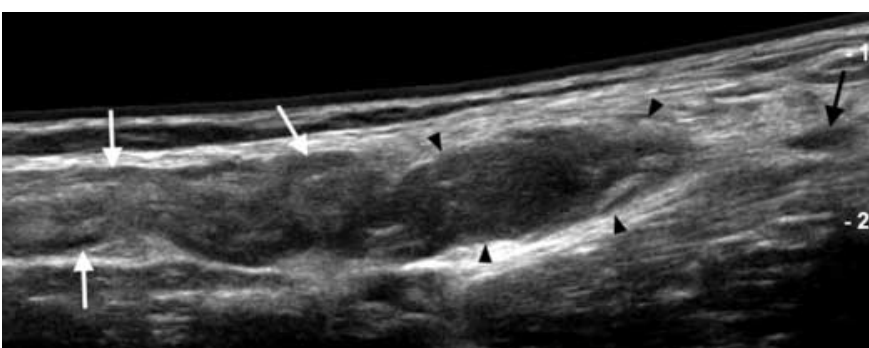

Abb. 1 35-jähriger Patient nach Stichverletzung vor 5 Wochen. Nach Durchtrennung des N. ulnaris kam es zur Ausbildung dieses scharf begrenzten, echoarmen, $1,2 \times 0,7 \mathrm{~cm}$ messenden Stumpfneuromes (Pfeilköpfe). Kontinuität zum proximalen Nervenende mit wellig verlaufenden, verplumpten Nervenfaszikeln (weiße Pfeile). Distal die regressiv veränderten Nervenabschnitte in der Nervenscheide echoarm nachweisbar (schwarzer Pfeil).

Fig. 135 year-old patient after stab-injury 5 weeks ago and penetration of N. ulnaris. Development of this circumscribed, hypoechoic neuroma, measuring $1.2 \times 0.7 \mathrm{~cm}$ (arrowheads). It is located in continuity with the proximal end of the injured nerve (white arrows). The distal stump consists of degenerated nerve components in surrounding nerve sheath (black arrow).

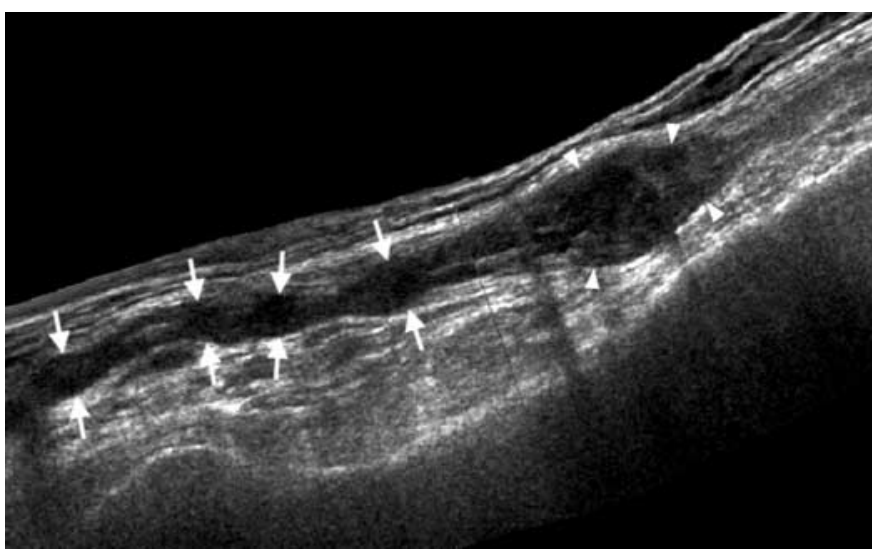

Abb. 2 Patient nach Verletzung des N. medianus im Bereich des Oberarmes. Im Längsschnitt werden im Verlauf des geschädigten Nervs mehrere Traktionsneurome sichtbar (Pfeile). Im Nervenstumpf kam es zur Ausbildung eines großen, umschriebenen Neuromes (Pfeilköpfe). Fig. 2 Patient after injury of N. medianus in the proximal upper extremity. In longitudinal sonogram there are multiple neuromas in the injured nerve itself (arrows). In the nerve stump there developed a bigger, hypoechoic, circumscribed neuroma (arrowheads).

\section{Morton-Neurom}

Äußerst schmerzhaft können Morton-Neurome sein, die im Rahmen degenerativer Veränderungen des N. digitalis plantaris communis an der Aufzweigung in die Nn. digitales plantares proprii entstehen. Sie liegen meist zwischen dem Os metatarsale III und IV, seltener zwischen dem Os metatarsale II und III. Ab einer Größe von etwa $5 \mathrm{~mm}$ werden sie klinisch relevant.

Charakteristisch für diese fusiformen Raumforderungen sind ödematöse Veränderungen und Fibrosierungen innerhalb des Nervs, wobei sich der Fibrosierungsprozess auch in das umgebende Weichteilgewebe ausdehnen kann [3, 5-8]. 
Sonographisch zeigen sich diese umschriebenen echoarmen Raumforderungen an typischer Lokalisation, sind rund im Transversalschnitt (Abb. 3) und spindelförmig im Längsschnitt.

\section{Nervenganglion}

Ebenfalls im Rahmen degenerativer Vorgänge kann es zur Ausbildung eines Nervenganglions kommen. Der betroffene Nerv weist eine lokalisierte Schwellung auf, bei der es sich um eine myxoide Veränderung mit zystischer Umwandlung handelt [3, $5-7]$.

Sonographisch lässt sich eine vorwiegend echoarme, septierte, zystische Formation mit in die Peripherie verlagerten Nervenfaszikeln nachweisen (Abb.4).

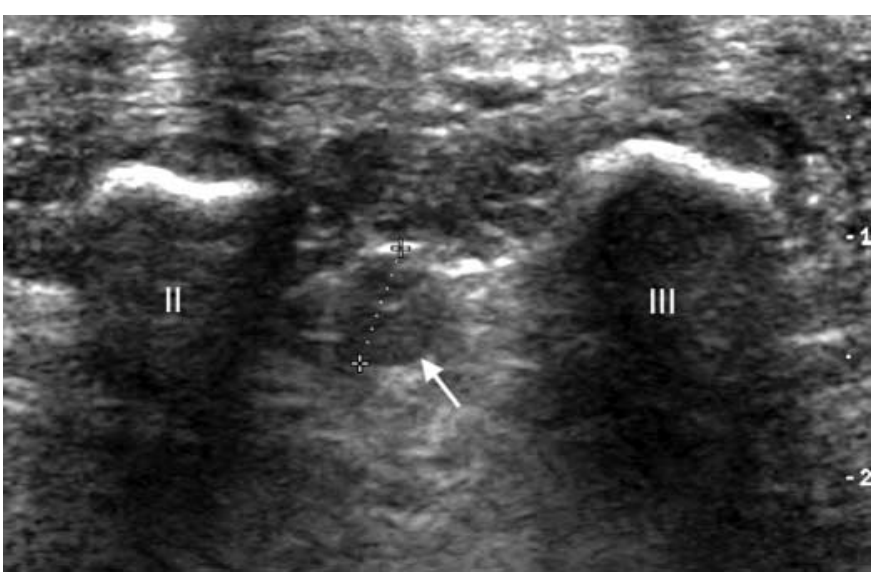

Abb. 3 48-jähriger Patient mit Schmerzen zwischen der zweiten und dritten Zehe. Zwischen dem Köpfchen des Os metatarsale II und III zeigt sich dieses scharf abgrenzbare, echoarme Morton-Neurom (weiBer Pfeil) mit einem Durchmesser von $5 \mathrm{~mm}$.

Fig. 348 year-old patient with pain between the second and third metatarsal head Sonographically there is seen this hypoechoic, circumscribed lesion measuring $5 \mathrm{~mm}$ in diameter, corresponding to morton's neuroma (white arrow).

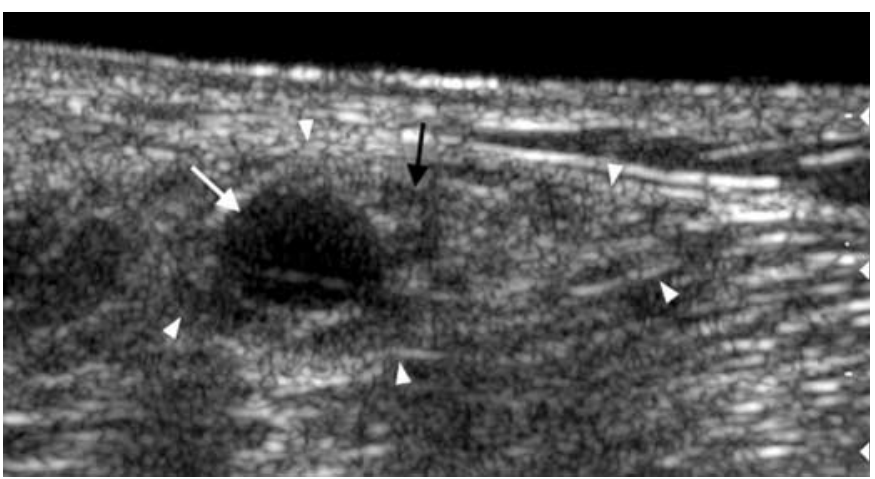

Abb. 4 Die Nervenfaszikel (schwarzer Pfeil) des N. peronaeus communis (Pfeilköpfe) werden durch ein umschriebenes, echoarmes, $0,8 \times 0,5 \mathrm{~cm}$ messendes Nervenganglion (weißer Pfeil) in die Peripherie verdrängt.

Fig. 4 Nerve fascicles (black arrow) of $\mathrm{N}$. peronaeus communis (arrowheads) are displaced by this well-defined, hypoechoic, cyst-like nerve sheath ganglion, measuring $0.8 \times 0.5 \mathrm{~cm}$ (white arrow).
Nervales Fibrolipom

Nervale Fibrolipome sind seltene Tumoren, die meist innerhalb der ersten 30 Lebensjahre diagnostiziert werden. Männer sind häufiger betroffen als Frauen. Das Fibrolipom der peripheren Nerven ist gekennzeichnet durch eine Infiltration des Peri- und Epineuriums durch fibröses Gewebe und Fettgewebe. Der betroffene Nerv kommt verplumpt zur Darstellung, durch das fibrolipöse Gewebe zeigt sich eine Separation der Nervenfaszikel.

Diese Raumforderungen wachsen langsam und können durch Kompression zu degenerativen Veränderungen des Nervs und dem Auftreten von Symptomen führen. In 30\% der Fälle zeigt sich ein Zusammenhang mit einer Makrodaktylie [5-8]. Sonographisch bieten die echoarmen Nervenfaszikel und das umgebende, isoechogene fibrolipöse Gewebe ein „kabelähnliches“ Erscheinungsbild (Abb. 5).

\section{Granulosazelltumor}

Granulosazelltumoren kommen meist als solitäre, schmerzlose Knoten zur Darstellung. Am häufigsten findet man sie in der Dermis oder Subkutis, aber auch in der Submukosa und in Muskelgewebe. In 10-15\% der Fälle zeigt sich ein multiples Auftreten. Granulosazelltumoren kommen meist bei Patienten in der 4 . bis 6. Lebensdekade vor, Kinder sind selten betroffen. Maligne Granulosazelltumoren sind äußerst selten.

Sonographisch stellen sich Granulosazelltumoren als gut abgrenzbare, zentral inhomogen echoarme, knotige Formationen dar. Häufig lassen sich im Inneren Verkalkungen nachweisen

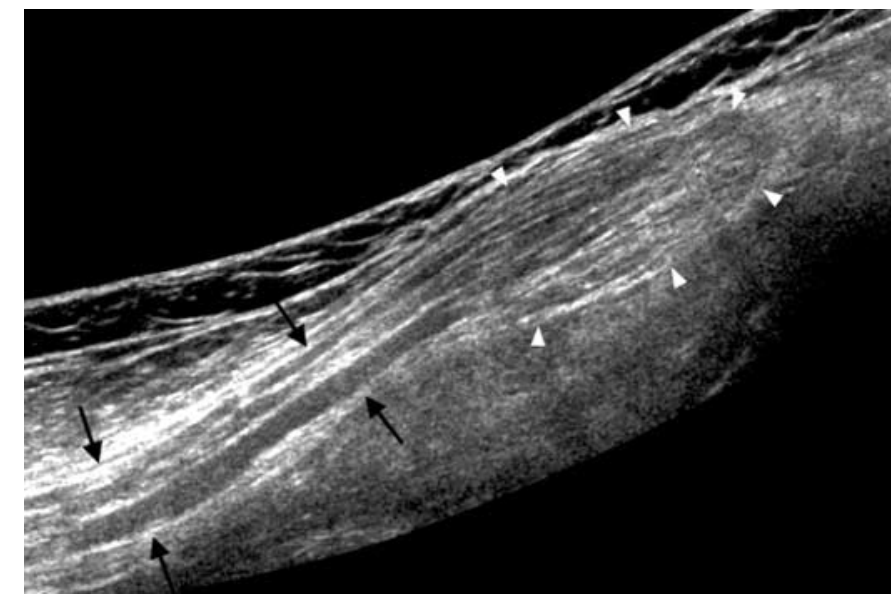

Abb. 5 37-jährige Patientin mit Raumforderung im Verlauf eines verplumpt zur Darstellung kommenden N. ischiadicus (schwarze Pfeile). Die Nervenfaszikel kommen separiert zur Darstellung durch dieses im Längsschnitt etwa $10 \mathrm{~cm}$ in größter Ausdehnung haltende nervale Fibrolipom (weiße Pfeilköpfe). Typisch das „kabelartige“ Erscheinungsbild.

Fig. 537 year-old patient with thickened N. ischiadicus (black arrows) that shows a neural fibrolipoma in its distal part (white arrowheads). Longitudinal sonogram shows a length of about $10 \mathrm{~cm}$. Cable-like, enlarged hypoechoic fascicles are separated by fatty, echogenic tissue. 


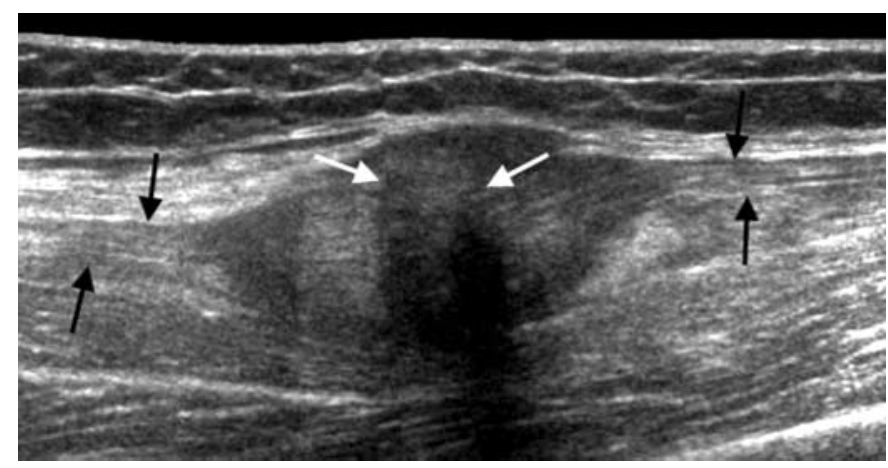

Abb. 6 Solitärer Granulosazelltumor bei einem 69-jährigen Patienten. Der Tumor geht kontinuierlich aus seinem Ursprungsnerv hervor (schwarze Pfeile), weist eine Ausdehnung von $3,5 \times 2,7 \mathrm{~cm}$ auf und ist gut abgrenzbar mit inhomogener Echotextur und Verkalkungen im Inneren (weiße Pfeile), die zu der vorhandenen dorsalen Schallauslöschung führen.

Fig. 6 Circumscribed granular-cell tumor with a size of $3.5 \times 2.7 \mathrm{~cm}$, heterogenous appearance and internal calcifications (white arrows). The tumor shows continuity with its nerve of origin (black arrows).

(Abb. 6). Farbdopplersonographisch lässt sich eine mäßige Vaskularisierung darstellen [5-7].

\section{Nervales Hämangiom}

Hämangiome, die sich innerhalb des Epineuriums entwickeln, sind äußerst seltene Tumoren. Sie müssen abgegrenzt werden von Hämangiomen, die den Nerv selbst erst sekundär miteinbeziehen (Abb. 7). Histologisch finden sich am häufigsten kavernöse Hämangiome ohne Hinweise auf Malignität.

Die Diagnostik lässt sich primär farbdopplersonographisch stellen $[5,7]$. Pathognomonisch sind die Präsenz von verkalkten Phlebolithen und der Pendelfluss des Blutes innerhalb der Gefäße bei Kompression des Tumors.

\section{Periphere Nervenscheidentumoren}

\section{Schwannom}

Schwannome sind langsam wachsende Tumoren, die meist exzentrisch im Verlaufe eines Nervs lokalisiert sind und von einer Kapsel, dem Epineurium, umgeben sind. Schwannome, die von großen Nerven ausgehen, sind außer entlang der Längsachse des Nervs frei beweglich. Bei ihrem Vorkommen in kleinen Nerven sind sie um einen zentralen Punkt frei beweglich, wobei hier häufig die Kontinuität zu ihrem Ursprungsnerv nicht erkennbar wird.

Histologisch finden sich die charakteristischen Antoni-A- und Antoni-B-Regionen. Die Antoni-A-Regionen weisen dicht arrangierte Spindelzellen auf, deren Zellkerne palisadenförmig angeordnet sind. Die Antoni-B-Regionen sind zellärmer und bestehen aus polymorphen Tumorzellen.

Schwannome weisen eine prominente Gefäßversorgung auf. Oft treten Thrombosen auf in diesen Gefäßen mit nachfolgenden Nekrosen und häufig entstehenden Blutungen.

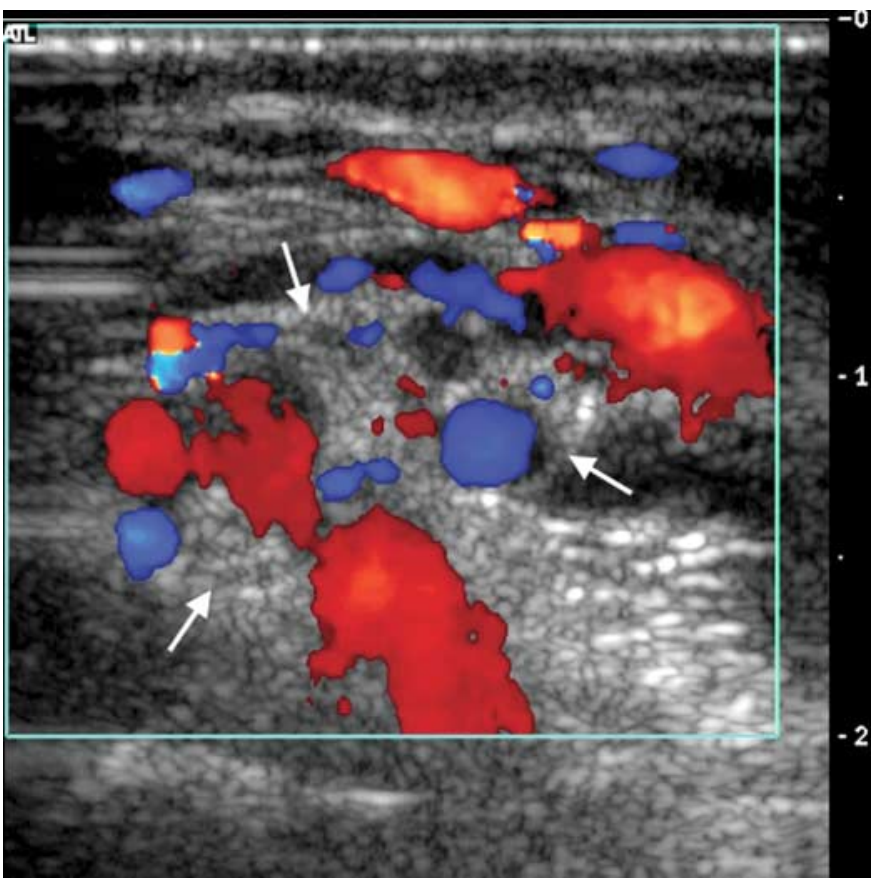

Abb. 7 40-jährige Patientin mit einem den N. medianus (Pfeile) vermutlich erst sekundär miteinbeziehenden Hämangiom. Der Nerv selbst zeigt einen Durchmesser von etwa $1,3 \mathrm{~cm}$. Farbdopplersonographisch die kavernös transformierten Gefäße sehr gut darstellbar.

Fig. 740 year-old patient with hemangioma that involves N. medianus (arrows). The nerve itself is about $1.3 \mathrm{~cm}$ in diameter. Colour Doppler sonography shows the cavernous vessels.

Neurologische Symptome und Schmerzen treten selten auf. Meist sind Patienten zwischen dem 20. und 50. Lebensjahr betroffen.

Sonographisch sind Schwannome runde oder ovale, gut umschriebene, fusiforme Raumforderungen mit echoarmer Binnenstruktur, die kontinuierlich aus ihrem Ursprungsnerv hervorgehen. Dieser zeigt eine unveränderte Echotextur [3-11]. Farbdopplersonographisch zeigt sich eine Hypervaskularisierung der Schwannome (Abb. 8, 9).

Eine eigene Untergruppe der Schwannome, die charakterisiert ist durch betonte degenerative Veränderungen, wird als degeneriertes Schwannom (engl. „ancient schwannoma“) bezeichnet. Zystische Veränderungen, Verkalkungen, auch Einblutungen dominieren das sonographische Bild. Dopplersonographisch sind auch sie immer gut vaskularisiert (Abb.10).

\section{Neurofibrom}

Neurofibrome sind ebenfalls langsam wachsende Tumoren, die im Nerv selbst gelegen sind. Sie sind umschrieben, in größeren Nerven von einer Kapsel umgeben, können aber, wenn sie von kleinen Nerven ausgehen, durch das Epineurium in das umgebende Weichteilgewebe infiltrieren, bleiben aber umschrieben. Neurofibrome fasern typischerweise die umgebenden Nervenfaszikel ihres Ursprungsnervs spindelförmig auf.

Neurofibrome verursachen selten Schmerzen und neurologische Symptome. Die betroffenen Patienten befinden sich meist in der 2. bis 3. Lebensdekade. 


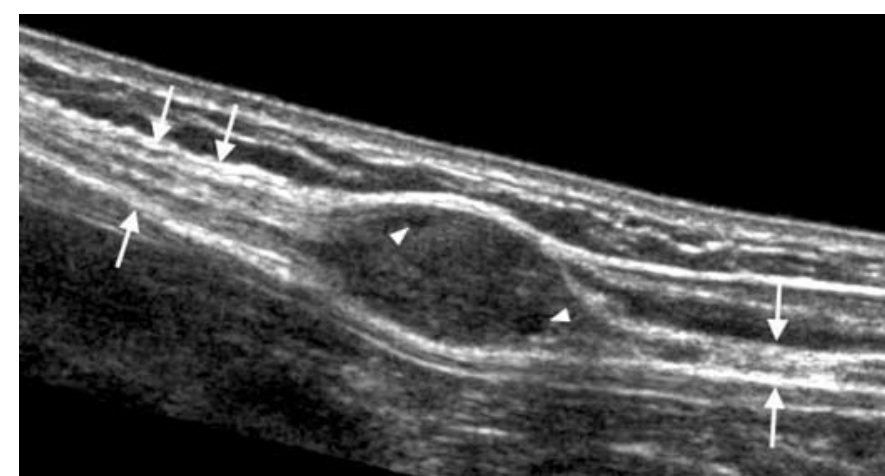

Abb. 8 46-jähriger Patient mit histologisch verifiziertem Schwannom des N. ulnaris, das kontinuierlich aus seinem Ursprungnerv hervorgeht (weiße Pfeile). Es stellt sich sonographisch gut umschrieben mit echoarmer Binnenstruktur dar und zwei kleinen Zysten im Inneren und misst 1,5 ×0,8 cm (Pfeilköpfe).

Fig. 846 year-old patient with histologically proven schwannoma of $\mathrm{N}$. ulnaris, measuring $1.5 \times 0.8 \mathrm{~cm}$. This circumscribed, hypoechoic tumor is located in continuity with normal appearing nerve of origin (white arrows) and shows two small internal cysts (arrowheads).

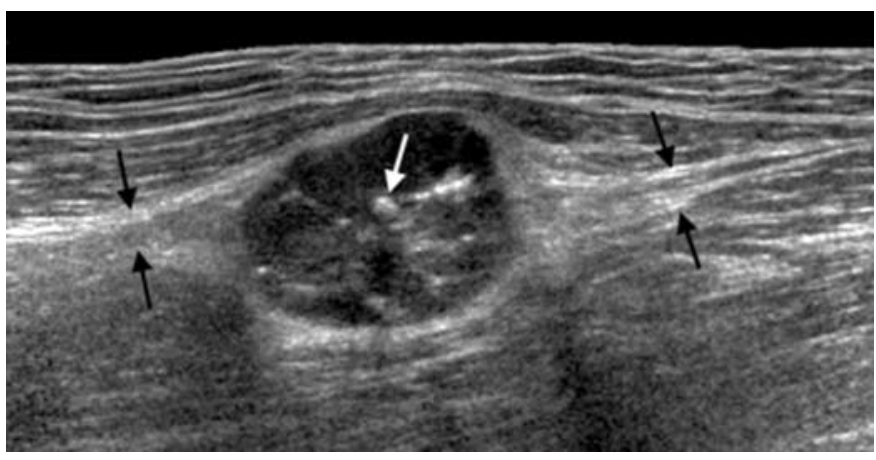

Abb. 9 Gut umschriebenes, echoarmes, $1,8 \times 2,2 \mathrm{~cm}$ messendes Schwannom mit Verkalkungen im Inneren (weißer Pfeil) im Verlauf eines Astes des N. femoralis. Auch hier gut erkennbar die Kontinuität zum Ursprungsnerv (schwarze Pfeile).

Fig. 9 Circumscribed, hypoechoic schwannoma with internal calcifications and a diameter of $1.8 \times 2.2 \mathrm{~cm}$ (white arrow). Normal entering and exiting branch of $\mathrm{N}$. femoralis (black arrows).

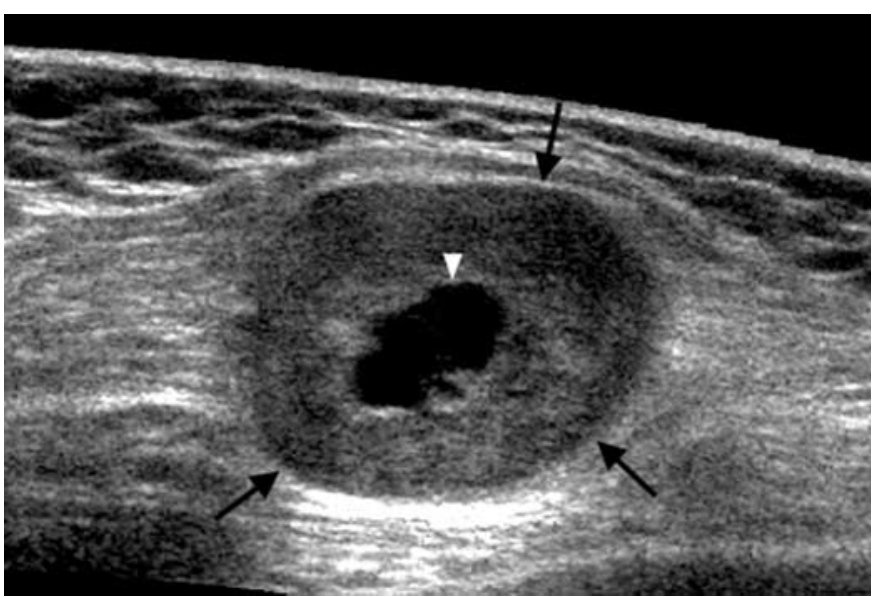

Abb. 10 75-jährige Patientin mit einem degenerierten Schwannom (schwarze Pfeile). Die gut umschriebene, 2,9 2 2,2 cm messende Raumforderung im Verlaufe eines Nervs innerhalb des M. latissimus dorsi zeigt zentral eine große zystische Veränderung (weißer Pfeilkopf).

Fig. 1075 year-old patient with an ancient schwannoma, $2.9 \times 2.2 \mathrm{~cm}$ in size. This hypoechoic, well-defined schwannoma (black arrows), located in continuity with its nerve of origin in the M. latissimus dorsi, shows a central hypoechoic region (white arrowhead).
Sonographisch wird ein konzentrisches Muster aus echoarmen und echoreichen Schichten beschrieben, das so genannte „target-sign“. Dieses entspricht dem mikroanatomischen Aufbau der Neurofibrome mit ihrer zentralen fibrokollagenösen Region und einer myxomatösen Peripherie. Farbdopplersonographisch sind sie eher gefäßarm [3-11].

Neben den solitär vorkommenden, lokalisierten Neurofibromen gibt es auch diffuse und plexiforme Neurofibrome.

Diffuse Neurofibrome treten meist in der Kindheit und bei jungen Erwachsenen auf. Sie sind schlecht abgrenzbar, infiltrieren das umgebende Gewebe, wobei dieses nicht zerstört wird, sondern durch das Tumorgewebe umgeben wird.

Plexiforme Neurofibrome findet man typischerweise im Rahmen der Neurofibromatose 1.

\section{Neurofibromatose 1}

Die Neurofibromatose 1 (NF1) oder der M. Recklinghausen ist eine Phakomatose mit einer Prävalenz von 1/2500-1/3000 Geburten.

Eine Neurofibromatose 1 ist gekennzeichnet durch das Auftreten multipler Neurofibrome (Abb. 11); es können die schon zuvor beschriebenen lokalisierten Neurofibrome auftreten, wobei diese meist tief gelegene, große Nerven betreffen und meist größer sind als bei solitärem Auftreten.

Nicht geklärt ist, wie oft diffuse Neurofibrome im Rahmen einer Neurofibromatose 1 auftreten.

Das plexiforme Neurofibrom ist eine Entität, die charakteristischerweise im Rahmen der Neurofibromatose 1 gefunden werden kann.

Plexiforme Neurofibrome entstehen fast immer in der frühen Kindheit. Sie sind meist große Läsionen, befallen ein langes Nervensegment und bilden multinoduläre Weichteilmassen (Abb.12).

Typisch für eine Neurofibromatose 1 ist weiters die Entwicklung von Café-au-Lait-Flecken an der Haut bei $90 \%$ der Patienten, weiters das Auftreten von Iris-Hamartomen und knöchernen Läsionen.

Zwischen 3 und 13\% der Patienten mit NF1 entwickeln einen malignen peripheren Nervenscheidentumor, üblicherweise nach einer Krankheitsdauer von 10 - 20 Jahren $[4$ - 7, 9, 10].

\section{Maligne periphere Nervenscheidentumoren}

Maligne periphere Nervenscheidentumoren gehen meistens von größeren Nerven aus und verursachen häufiger neurologische Symptome als benigne Tumoren peripherer Nerven.

Die Tumorzellen formieren sich zu Faszikeln, zusätzlich zeigen sich oft Einblutungen und nekrotische Areale. Weiters können auch Ossifikationen und Verkalkungen vorkommen sowie glanduläre und muskuläre Komponenten. Sie können gut umschrieben oder auch multinodulär zur Darstellung kommen (Abb.13,14). 


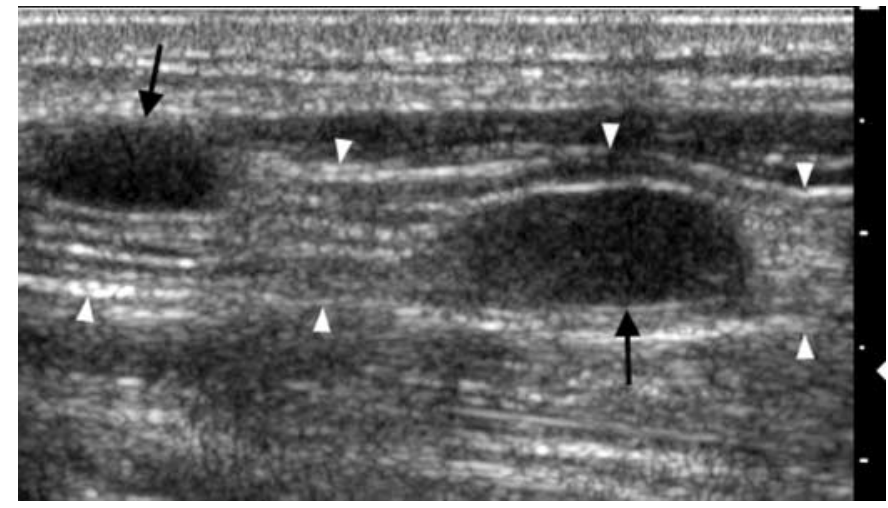

Abb. 11 33-jähriger Patient mit bekannter Neurofibromatose 1 und zwei echoarmen Neurofibromen (schwarze Pfeile), die kontinuierlich aus ihrem Ursprungsnerv (weiße Pfeilköpfe) hervorgehen. Die Ausdehnung des kleineren Neurofibromes beträgt $0,8 \times 0,3 \mathrm{~cm}$, die des größeren $1,3 \times 0,5 \mathrm{~cm}$.

Fig. 1133 year-old patient with neurofibromatosis 1 and two hypoechoic neurofibromas (black arrows) with entering and exiting nerve of origin (white arrowheads). The neurofibromas are $0.8 \times 0.3 \mathrm{~cm}$ and $1.3 \times 0.5 \mathrm{~cm}$ in diameter.

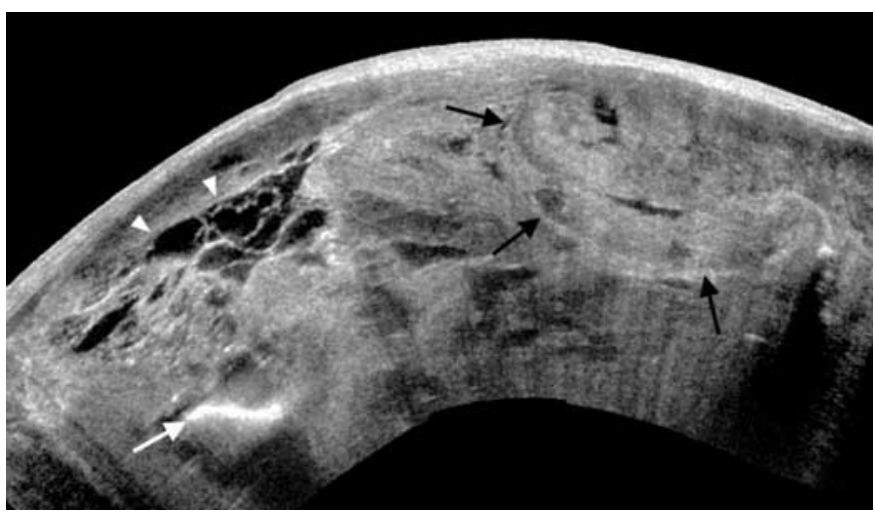

Abb. 13 36-jähriger Patient mit bekannter Neurofibromatose und diesem malignen peripheren Nervenscheidentumor im Bereich der rechten Schulter. Im Inneren des Tumors ausgedehnte zystisch-nekrotische Areale (weiße Pfeilköpfe) sowie Verkalkungen (weißer Pfeil). Noduläre Weichteilmasse im Inneren des Tumors (schwarze Pfeile). Die Gesamtausdehnung des Tumors beträgt etwa $18 \mathrm{~cm}$.

Fig. 1336 year-old patient with neurofibromatosis 1 and this malignant peripheral nerve sheath tumor at the right shoulder, measuring about $18 \mathrm{~cm}$ in length. Internal extensive cystic and necrotical areas (white arrowheads) and calcifications (white arrow). Nodular tumorous region (black arrows).

Maligne periphere Nervenscheidentumoren wachsen lokal infiltrierend und können metastasieren. Dies vor allem in Lunge, Leber, Subkutis und Knochen. Sie neigen nach Resektion zur Bildung von Lokalrezidiven $[5-7,9,10]$.

\section{Literatur}

${ }^{1}$ Fornage BD. Peripheral nerves of the extremities: imaging with ultrasound. Radiology 1988; 167: 179-182

2 Riede UN, Schaefer HE. Allgemeine und spezielle Pathologie. Stuttgart, New York: Georg Thieme Verlag, 1995

${ }^{3}$ Bacigalupo L, Bianchi S, Valle M et al. Ultraschall peripherer Nerven. Radiologe 2003; 43: 841 - 849

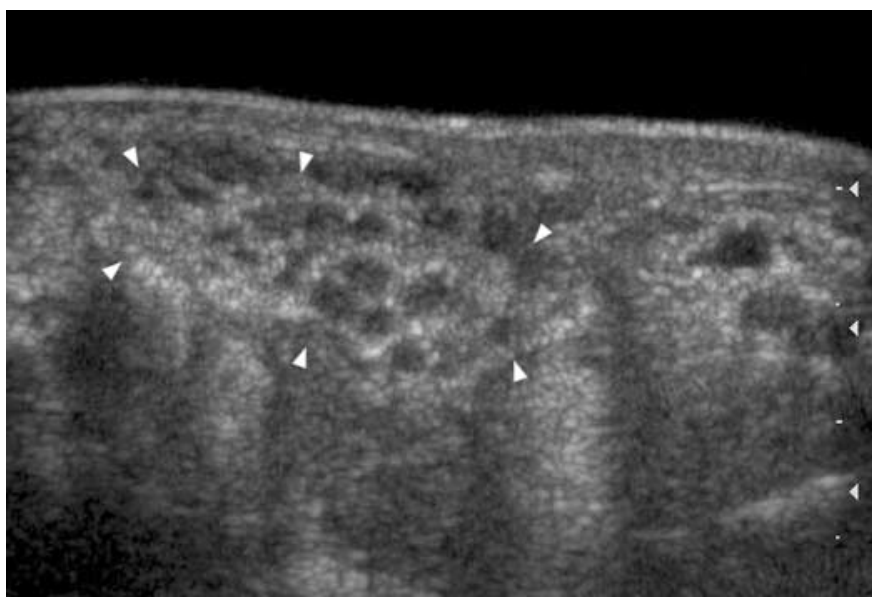

Abb. 12 Stark aufgetriebener, etwa $1,6 \times 0,6 \mathrm{~cm}$ messender N. medianus einer 7-jährigen Patientin (Pfeilköpfe). Zwischen den gut erkennbaren Nervenfaszikeln zeigt sich im Transversalschnitt vermehrt fibröses Stroma. Es handelte sich bei dieser Raumforderung um ein plexiformes Neurofibrom.

Fig. 12 Thickened $\mathrm{N}$. medianus of a 7 year-old patient, measuring about $1.6 \times 0.6 \mathrm{~cm}$. (arrowheads). Transverse sonogram shows hypoechoic nerve fascicles and surrounding tissue of a plexiform neurofibroma of N. medianus.

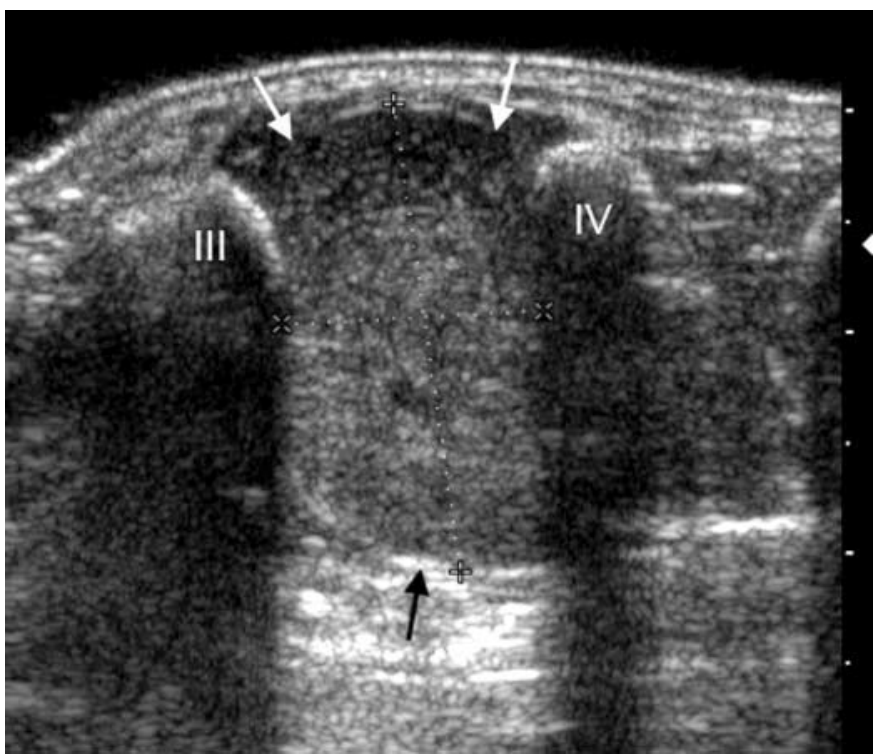

Abb. 14 Auf Höhe des dritten bzw. vierten Metakarpalknochens zeigte sich bei diesem 42-jährigen Patienten diese knapp $2 \times 3 \mathrm{~cm}$ messende, gut abgrenzbare Raumforderung (Pfeile), die farbdopplersonographisch aufgrund der anarchischen Gefäßarchitektur und hohen Flussgeschwindigkeiten suspekt erschien. Nach histologischer Aufarbeitung entnommener Proben ergab sich die Diagnose eines malignen peripheren Nervenscheidentumors.

Fig. 1442 year-old patient with circumscribed tumor between third and fourth metacarpal, measuring $2 \times 3 \mathrm{~cm}$ (arrows). Suspect signals in colour doppler sonography and architecture of vessels led to biopsy and the diagnosis of malignant peripheral nerve sheath tumor.

${ }^{4}$ Chiou HJ, Chou YH, Chiou SY et al. Peripheral nerve lesions: Role of high-resolution ultrasound. Radiographics 2003; 23: 15e

${ }^{5}$ Enzinger FM, Weiss SW. Soft Tissue Tumors. Mosby-Year book. 3rd edition. St. Louis: Mosby, 1995

${ }^{6}$ Murphey MD, Smith WS, Smith SE et al. Imaging of musculosceletal neurogenic Tumors: Radiologic-pathologic Correlation. Radiographics 1999; 19: $1253-1280$ 
${ }^{7}$ Peer S, Bodner G. High-resolution Sonography of the Peripheral Nervous System. Berlin, Heidelberg, New York: Springer-Verlag, 2003

${ }^{8}$ Stuart RM, Koh ESC, Breidahl WH. Sonography of peripheral nerve pathology. AJR 2003; 182: 123

${ }^{9}$ De Schepper AM. Imaging of Soft Tissue Tumors. Berlin, Heidelberg: Springer-Verlag, 1997
${ }^{10}$ Lin J, Martel W. Cross-sectional imaging of peripheral nerve sheath tumors. AJR 2001; 176: 75

${ }^{11}$ Reynolds DL, Jacobson JA, Inampudi P et al. Sonographic characteristics of peripheral nerve sheath tumors. AJR 2004; 182: 741 - 744 